\title{
SeqEnhDL: sequence-based classification of cell type-specific enhancers using deep learning models
}

Yupeng Wang ${ }^{1,3^{*}} \mathbb{D}$, Rosario B. Jaime-Lara ${ }^{2,3}$, Abhrarup Roy ${ }^{3}$, Ying Sun ${ }^{1}$, Xinyue Liu ${ }^{1}$ and Paule V. Joseph $2,3^{*}$

\begin{abstract}
Objective: To address the challenge of computational identification of cell type-specific regulatory elements on a genome-wide scale.

Results: We propose SeqEnhDL, a deep learning framework for classifying cell type-specific enhancers based on sequence features. DNA sequences of "strong enhancer" chromatin states in nine cell types from the ENCODE project were retrieved to build and test enhancer classifiers. For any DNA sequence, positional $k$-mer $(k=5,7,9$ and 11$)$ fold changes relative to randomly selected non-coding sequences across each nucleotide position were used as features for deep learning models. Three deep learning models were implemented, including multi-layer perceptron (MLP), Convolutional Neural Network (CNN) and Recurrent Neural Network (RNN). All models in SeqEnhDL outperform stateof-the-art enhancer classifiers (including gkm-SVM and DanQ) in distinguishing cell type-specific enhancers from randomly selected non-coding sequences. Moreover, SeqEnhDL can directly discriminate enhancers from different cell types, which has not been achieved by other enhancer classifiers. Our analysis suggests that both enhancers and their tissue-specificity can be accurately identified based on their sequence features. SeqEnhDL is publicly available at https://github.com/wyp1125/SeqEnhDL.
\end{abstract}

Keywords: Enhancer, Classification, Deep learning, DNA sequence, Cell type

\section{Introduction}

Cell type-specific enhancers, cis-regulatory elements that up-regulate gene transcription in a cell type, play a key role in determining the regulatory landscape of the human genome [1]. Enhancers are commonly located in the introns and immediately upstream of their target genes' transcription start site (TSS). They are also known to populate gene deserts [2], reside in introns of neighboring genes [3], and co-localize with coding exons [4]. Enhancer mutations are often associated with diseases [5-7]. Accurate prediction of enhancers from DNA

*Correspondence: ywang@bdxconsult.com; paule.joseph@nih.gov

${ }^{3}$ Division of Intramural Research, National Institute of Nursing Research,

National Institutes of Health, Bethesda, MD 20892, USA

Full list of author information is available at the end of the article sequences is the basis of assessing whether mutation(s) can disrupt an enhancer's activity, a type of mechanism for genetic diseases.

Predicting enhancers based on transcription factor binding sites (TFBS) was proposed because TFBS tend to be conserved over vertebrate evolution [8-10]. However, there is uncertainty regarding the identification of TFBS from DNA sequences. To ameliorate this challenge, direct sequence features such as $k$-mers (i.e., nucleotide sequences with a specified length) were then introduced to model enhancer prediction [11, 12]. However, these early studies did not achieve high prediction accuracy nor were they able to distinguish enhancers of different cell types.

With the wide application of ChIP-seq technologies, enhancers were frequently profiled on a genome-wide

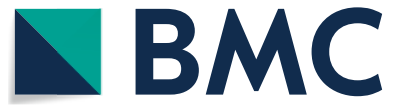

(c) The Author(s) 2021. This article is licensed under a Creative Commons Attribution 4.0 International License, which permits use, sharing, adaptation, distribution and reproduction in any medium or format, as long as you give appropriate credit to the original author(s) and the source, provide a link to the Creative Commons licence, and indicate if changes were made. The images or other third party material in this article are included in the article's Creative Commons licence, unless indicated otherwise in a credit line to the material. If material is not included in the article's Creative Commons licence and your intended use is not permitted by statutory regulation or exceeds the permitted use, you will need to obtain permission directly from the copyright holder. To view a copy of this licence, visit http://creativeco mmons.org/licenses/by/4.0/. The Creative Commons Public Domain Dedication waiver (http://creativecommons.org/publicdomain/ zero/1.0/) applies to the data made available in this article, unless otherwise stated in a credit line to the data. 
scale [13]. The ENCODE project produced genomewide profiles of various epigenetic marks for multiple human cell types [14]. By applying a hidden Markov model (i.e. ChromHMM) to these epigenetic marks, the human genome sequence has been binned into more than ten chromatin states, including enhancers $[15,16]$. The "strong enhancer" state, shown to be associated with increased gene expression, provides genome-wide positioning of active enhancers specific to a cell type [15]. Although these datasets' availability renders enhancers' positioning unnecessary, enhancers' sequence structures, especially their subtle differences among cell types, can help understand cell type-specific gene regulation and should be explored.

The proper generation of negative sequences influences the effectiveness of enhancer classifiers. Negative sequences should contain similar basic sequence features with enhancers such as length distributions, GC, and repeat contents $[12,17,18]$; otherwise, enhancer classifiers may learn different nucleotide compositions rather than occurrences of key DNA motifs. Although many studies reported sequence-based enhancer prediction, it is still unknown whether enhancers can be distinguished between different cell types or tissues based on sequences.

The sequence structures of enhancers may not be linear or additive. In fact, there could be complex grammar or semantics among different DNA elements of an enhancer $[19,20]$. In recent years, deep learning technologies have gained greater popularity compared to conventional machine learning methods, and have been adapted in biomedical research to address complex research questions [21-29]. Thus, deep learning can be more powerful in classifying enhancers. In this study, we propose SeqEn$\mathrm{hDL}$, a deep learning framework for the classification of cell type-specific enhancers based on sequence features. To include interdependency and sequence information in the features of a DNA sequence, SeqEnhDL uses positional $k$-mer fold changes across each nucleotide position as its features. The effectiveness and advantages of SeqEnhDL are demonstrated based on the chromatin state segmentation data of nine cell types from the ENCODE project [14].

\section{Main text \\ Methods}

\section{Genome annotations}

The sequences and transcripts of the human genome (hg19) were obtained from the UCSC genome browser. The "knowngene" dataset was used to guide masking exons. Chromatin state annotations of gm12878, H1hesc, hepg2, Hmec, Hsmm, Huvec, K562, Nhek, and Nhlf cell types generated by ChromHMM [30] were obtained from the ENCODE project (Broad version). The data included a total of 15 chromatin states. 4_Strong_Enhancer and 5_Strong_Enhancer states were used as enhancers in this study.

\section{Detailed computational procedure}

See Additional file 1: methods.

\section{Results}

\section{The SeqEnhDL framework}

The SeqEnhDL framework is depicted in Fig. 1a. The framework started from "bed" files containing the chromosomal positions of a large number (e.g. > 1000) of enhancers for a cell type. The DNA sequences of enhancers were retrieved from the human genome where exon and repetitive sequences were masked. These DNA sequences were then divided into individual enhancers with a fixed length of $200 \mathrm{bp}$, which makes features more standardized and comparable. 200 bp is recommended because it corresponds with the resolution of a nucleosome and spacer region, though other lengths can be used. Enhancer sequences were used as the positive sequences. Control sequences for computing $k$ mer fold changes, and negative sequences for testing enhancer classifiers, were randomly selected from the genome where exon, repetitive and enhancer sequences were masked, according to the GC contents of enhancer sequences. $K$-mer $(k=5,7,9$, and 11$)$ fold changes between all enhancer and control sequences of a cell type were computed and used as dictionaries. To convert a DNA sequence to features, $k$-mer $(k=5,7,9$, and $11)$ fold changes at each nucleotide position, referred to as positional $k$-mer fold changes, were generated according to the dictionaries of the cell type. Of note, we chose odd $k$-mers because fold changes of different $k$-mers can be aligned at their central nucleotide position. Then, features of each nucleotide position of a DNA sequence were concatenated, resulting in a $200 \times 4$ array of features for that DNA sequence. An intuitive example for the feature extraction process is shown in Fig. 1b. Thus, our feature extraction process retained interdependency and sequence information among the nucleotide positions of a DNA sequence.

Any dataset for building a deep learning enhancer classifier should be divided into training, validation, and testing data, in a cross-validation mode (e.g., 5-fold). Evaluation metrics were based on the average of each fold of cross-validation. Three deep learning models-MLP, $\mathrm{CNN}$, and RNN, were built. Multilayer perceptrons are fully connected networks. CNN takes advantage of the hierarchical pattern in data and assembles more complex patterns using smaller and simpler patterns. RNN makes use of sequential information among features. 


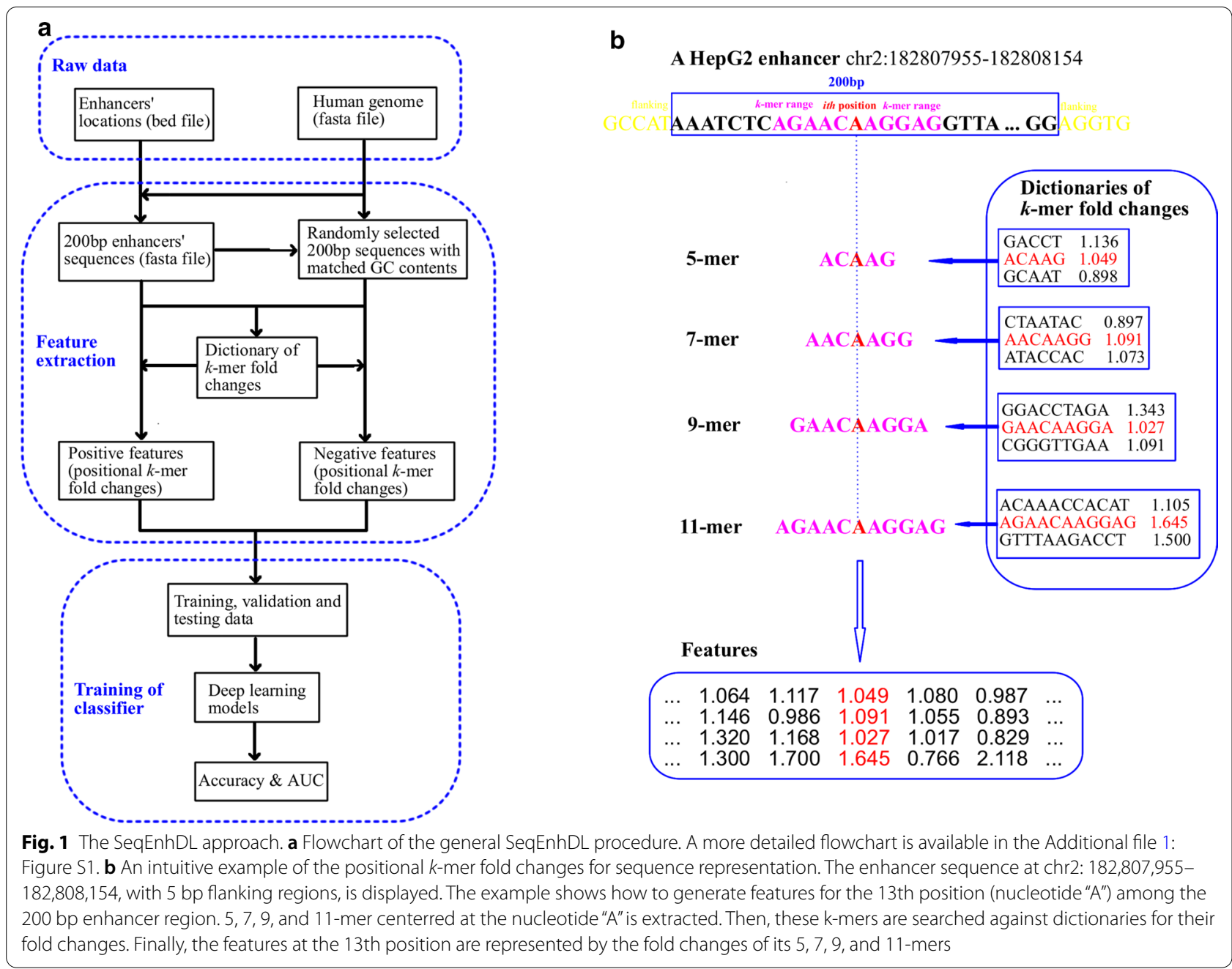

Particularly, bidirectional long short-term memory (LSTM) RNN, which can learn long-term dependencies, was adopted. Because we adopted the positional $k$-mer fold changes, which utilize nucleotide position indexing, features at different positions (especially adjacent positions) could be interdependent. In addition, different $k$-mers $[5,7,9,11]$ at a nucleotide position represented the second dimension of features. The interdependency and sequence information of positional $k$-mer fold changes among nucleotide positions rationalize the use of CNN and RNN architectures.

\section{Evaluation of the performance of SeqEnhDL}

Discriminating enhancers of a single cell type/tissue from randomly selected sequences have been studied before and provided the foundation for evaluating the performance of SeqEnhDL. We retrieved DNA sequences located within the "strong enhancers" chromatin states of nine cell types from the ENCODE project [14]. The performance of SeqEnhDL was evaluated in terms of accuracy and area under the curve (AUC) for distinguishing enhancers in each cell type. Stateof-the-art methods were selected for comparison with SeqEhnDL. gkm-SVM [12, 17] was chosen for comparison because it uses $k$-mer information to predict enhancers. DanQ [28] was chosen for comparison because it is an RNN-based tool for predicting the functions of noncoding sequences. The performance of DanQ on each cell type was represented by the highest statistics among predictions on 919 ChIP/DNase-seq marks. When different tools were executed, five-fold cross-validation was employed in order to generate reliable performance measures. Comparisons of performances among different tools (Fig. 2) show that all the three models of SeqEnhDL greatly outperform gkm-SVM and DanQ. SeqEnhDL's accuracies range from 0.961 to 0.999 , suggesting that enhancers can be accurately identified on different cell types. Comparison of Receiver Operating Characteristic (ROC) curves on the hepg2 cell type (Additional file 1: Figure 

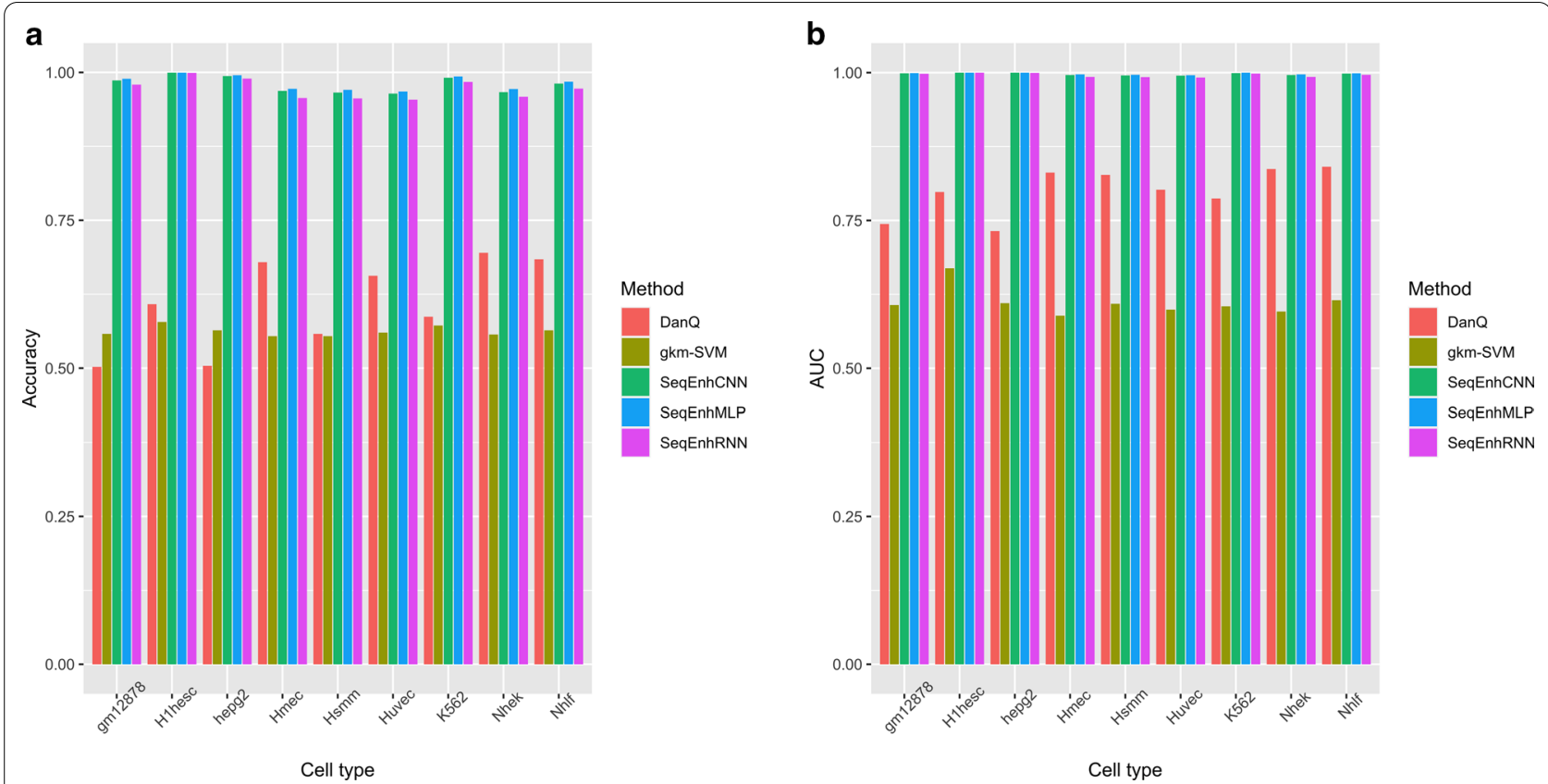

Fig. 2 Comparison among different enhancer classifiers with regard to distinguishing cell type-specific enhancers from randomly selected non-coding sequences. a Comparison of accuracies. $\mathbf{b}$ Comparison of AUCS

S2) re-confirmed that SeqEnhDL performed better. Of note, we also ran a recent approach based on the ensemble of deep RNNs [29] for a comparison. However, its accuracies and AUCs were around 0.5 (Additional file 1: Table S1), indicating that this compared approach was ineffective on this study's datasets.

To validate the advantages of deep learning models over conventional machine learning models regarding enhancer classification, we flattened the $k$-mer features. We built enhancer classifiers based on six conventional machine learning models. Note that 2000 positive and negative sequences were randomly selected for each cell type and repeated ten times to ensure training each deep learning and conventional machine learning model could be finished within $1 \mathrm{~h}$. Additional file 1: Figure S3 shows that accuracies of SeqEnhCNN and SeqEnhRNN are consistently higher than conventional machine learning models, and SeqEnhMLP is among the second tier in most cell types. These analyses collectively suggest that enhancers present in a single cell type can be accurately identified based on sequence features by SeqEnhDL, and SeqEnhDL significantly outperforms existing methods by better discriminating enhancers from randomly selected sequences.

\section{SeqEnhDL can discriminate enhancers' cell types based on DNA sequences}

Successful machine learning models for distinguishing enhancers from different cell types must learn celltype-specific sequence structures such as domains, motifs, and their interactions. Previous enhancer classifiers were not examined regarding this capacity. Some may be adapted for distinguishing enhancers from different cell types by treating one cell type as the negative group. We applied gkm-SVM and SeqEnhDL to distinguish enhancers from different cell types. We switched the assignments of positive and negative groups for each pair of cell types and computed the average accuracy and AUC. The accuracies and AUCs for all pairs of cell types are displayed in Fig. 3. The accuracies and AUCs of gkm-SVM for all pairs of cell types are around 0.5 , indicating that gkm-SVM failed to capture tissuespecificities of enhancers. In contrast, all models of SeqEnhDL generated high accuracies (e.g. $>0.9$ ) and AUCs (e.g. > 0.95) in most cell type combinations, indicating that SeqEnhDL can identify tissue-specificity. This analysis suggests that SeqEnhDL can learn complex sequence features related to tissue-specificities and discriminate enhancers from different cell types. 

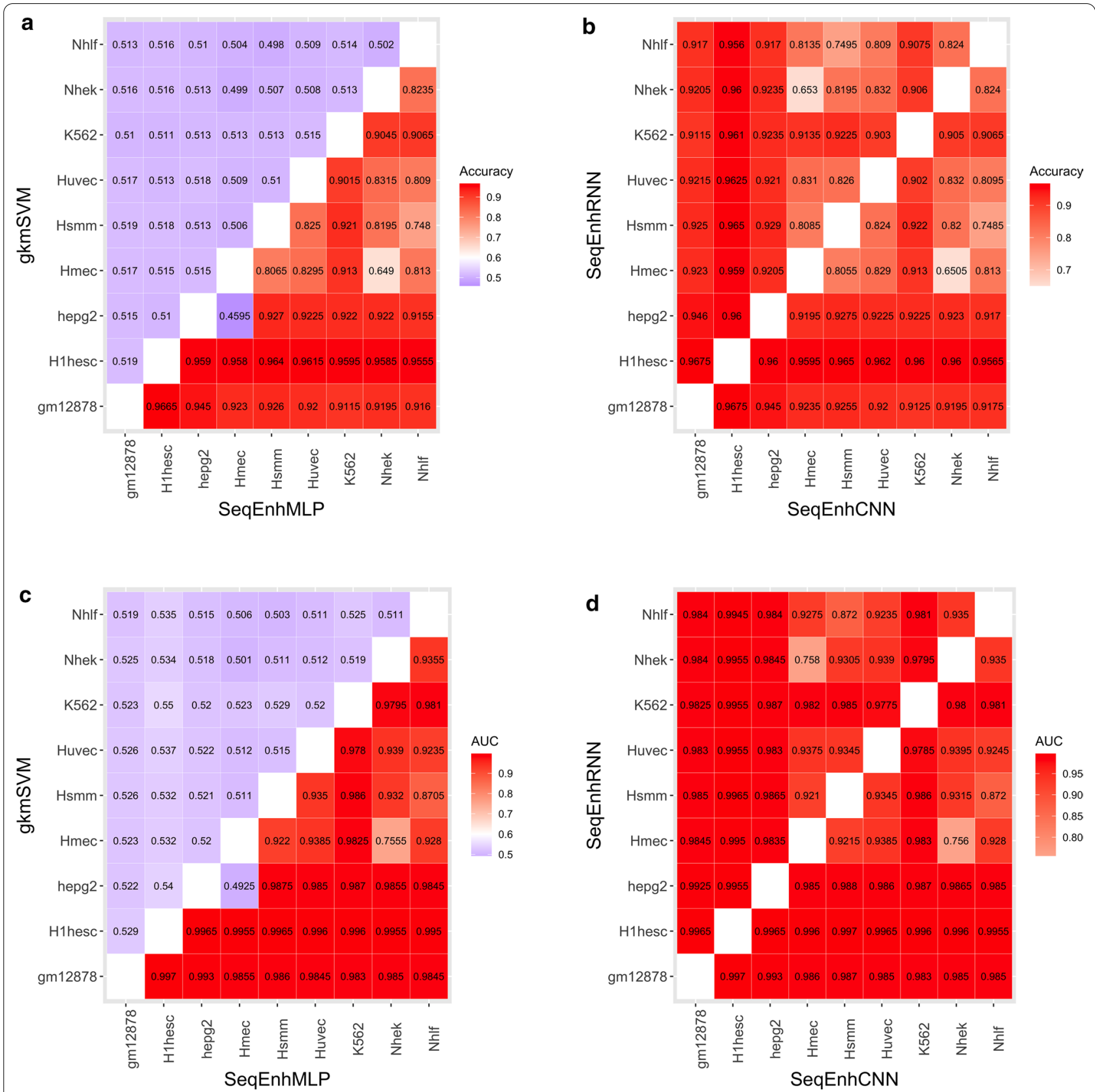

Fig. 3 Comparison between gkm-SVM and SeqEnhDL with regard to discriminating enhancers from two cell types. a, b Comparison of accuracies. c, $\mathbf{d}$ Comparison of AUCs

\section{Discussion}

When we built enhancer classifiers, we separated control and negative sequences. We used equal numbers of positive and negative sequences, which can significantly reduce the chances of overfitting, a common machine learning problem. We used all enhancers' sequences to compute $k$-mer fold changes. Although theoretically, enhancers can be divided into two subsets (one for computing $k$-mer fold changes and the other for testing enhancer classifiers), it has practical limitations because longer $k$-mers are very important for composing enhancers and may occur only a few times in a cell type.

We successfully applied SeqEnhDL to discriminate enhancers from two cell types. gkm-SVM failed to distinguish enhancers from different cell types, indicating that most (if not all) previous $k$-mer based models tend 
to learn the common features of enhancers rather than tissue-specific motif structures. This successful application suggests that tissue/cell type-specific gene regulation could be better understood based on machine learning of high-level enhancers' structures.

SeqEnhDL adopts positional $k$-mer fold changes as the sequence representation. This representation utilizes nucleotide positions, rather than $k$-mer indices. Positional $k$-mer fold changes reflect the degrees of k-mers' specificity to a tissue/cell type regardless of the exact sequence. These positional $k$-mer fold changes increase the likelihood of capturing complex and high-level sequence features, which may aid the performance of SeqEnhDL.

Over a 200 bp sequence, important $k$-mers could appear at any position, and any important local patterns should be captured and contribute to enhancer prediction. Compared with conventional machine learning models, deep learning models extract high-level patterns from the features. Thus, it is not practical or reasonable to discriminate which nucleotide positions are more important than others when sequences are represented using positional $k$-mer fold changes.

\section{Conclusion}

We propose SeqEnhDL, a feature extraction and deep learning framework for classifying cell-type-specific enhancers based on sequence features. A variety of analyses were performed to demonstrate that SeqEnhDL outperforms existing enhancer classifiers. We further proved that SeqEnhDL could be used to discriminate enhancers from different cell types.

\section{Limitation}

The training dataset of this study from ChromHMM may be highly noisy. The primary goal of this study is to demonstrate the effectiveness of SeqEnhDL. SeqEnhDL is expected to perform better on cleaner datasets. Specific high-level features that are important for enhancer classification have not been addressed in this study and remain an open question.

\section{Supplementary Information}

The online version contains supplementary material available at https://doi. org/10.1186/s13104-021-05518-7.

Additional file 1: Table S1. Accuracies and AUCs of the ensemble of deep RNN approach. Table S2. Number of enhancers in each cell type after the filtering procedure. Table S3. Structures of deep learning models in SeqEnhDL. Table S3. Parameters of conventional machine learning models. Figure S1. Flowchart of the detailed SeqEnhDL procedure. Figure S2. Comparison among different enhancer classifiers in terms of ROC curves. Figure S3. Comparison between SeqEnhDL and conventional machine learning models.
Acknowledgements

The authors would like to thank Dr. Joan Austin for her comments and editorial assistance.

\section{Authors' contributions}

YW and PVJ conceived the study. YW, YS and XL made the programs. YW, PVJ, RJL and AR performed the analyses. YW and PVJ were primarily responsible for preparation of the manuscript. All authors read and approved the final manuscript.

\section{Funding}

Open Access funding provided by the National Institutes of Health (NIH). PVJ is supported by the National Institute of Nursing Research [1ZIANR000035-01]. PVJ is also supported by the Office of Workforce Diversity, National Institutes of Health and the Rockefeller University Heilbrunn Nurse Scholar Award[no award number]. RBJL received Intramural Research Training Awards, Office of Intramural Training and Education, National Institutes of Health, Department of Health and Human Services[no award number]. RBJL received NIH Center on Compulsive Behaviors Fellowship Award. Funding for open access charge: National Institutes of Health.

\section{Availability of data and materials}

Genome sequences and annotations were downloaded from UCSC genome browser (http://genome.ucsc.edu/). Chromatin state segmentation data were downloaded from the ENCODE project (http://hgdownload.soe.ucsc.edu/ goldenPath/hg19/encodeDCC/wgEncodeBroadHmm/). Programs of this study were written in Perl, Python and R. All source code is freely available at https://github.com/wyp1 125/SeqEnhDL. Testing datasets of SeqEnhDL for reproduction purpose are available at http://www.bdxconsult.com/SeqEnhDL. Supplementary methods, tables and figures are available in Additional file 1.

\section{Declarations}

Ethics approval and consent to participate Not applicable.

Consent for publication

Not applicable.

Competing interests

Not applicable.

\section{Author details}

${ }^{1}$ BDX Research and Consulting LLC, Herndon, VA 20171, USA. ${ }^{2}$ Division of Intramural Clinical and Biological Research (DICBR), National Institute on Alcohol Abuse and Alcoholism, National Institutes of Health, Bethesda, MD 20892, USA. ${ }^{3}$ Division of Intramural Research, National Institute of Nursing Research, National Institutes of Health, Bethesda, MD 20892, USA.

Received: 12 October 2020 Accepted: 9 March 2021

Published online: 19 March 2021

\section{References}

1. Maston GA, Evans SK, Green MR. Transcriptional regulatory elements in the human genome. Annu Rev Genomics Hum Genet. 2006;7:29-59.

2. Nobrega MA, Ovcharenko I, Afzal V, Rubin EM. Scanning human gene deserts for long-range enhancers. Science. 2003;302:413.

3. Ott CJ, Suszko M, Blackledge NP, Wright JE, Crawford GE, Harris A. A complex intronic enhancer regulates expression of the CFTR gene by direct interaction with the promoter. J Cell Mol Med. 2009;13:680-92.

4. Birnbaum RY, Clowney EJ, Agamy O, Kim MJ, Zhao J, Yamanaka T, Pappalardo Z, Clarke SL, Wenger AM, Nguyen L, et al. Coding exons function as tissue-specific enhancers of nearby genes. Genome Res. 2012;22:1059-68.

5. Weedon MN, Cebola I, Patch AM, Flanagan SE, De Franco E, Caswell R, Rodriguez-Segui SA, Shaw-Smith C, Cho CH, Allen HL, et al. Recessive mutations in a distal PTF1A enhancer cause isolated pancreatic agenesis. Nat Genet. 2014;46:61-4. 
6. Emison ES, McCallion AS, Kashuk CS, Bush RT, Grice E, Lin S, Portnoy ME, Cutler DJ, Green ED, Chakravarti A. A common sex-dependent mutation in a RET enhancer underlies Hirschsprung disease risk. Nature. 2005;434:857-63.

7. Pasquali L, Gaulton KJ, Rodriguez-Segui SA, Mularoni L, Miguel-Escalada I, Akerman I, Tena JJ, Moran I, Gomez-Marin C, van de Bunt M, et al. Pancreatic islet enhancer clusters enriched in type 2 diabetes risk-associated variants. Nat Genet. 2014;46:136-43.

8. Taher L, McGaughey DM, Maragh S, Aneas I, Bessling SL, Miller W, Nobrega MA, McCallion AS, Ovcharenko I. Genome-wide identification of conserved regulatory function in diverged sequences. Genome Res. 2011:21:1139-49.

9. Narlikar L, Sakabe NJ, Blanski AA, Arimura FE, Westlund JM, Nobrega MA, Ovcharenko I. Genome-wide discovery of human heart enhancers. Genome Res. 2010;20:381-92.

10. Burzynski GM, Reed X, Taher L, Stine ZE, Matsui T, Ovcharenko I, McCallion AS. Systematic elucidation and in vivo validation of sequences enriched in hindbrain transcriptional control. Genome Res. 2012;22:2278-89.

11. Lee $D$, Karchin $R$, Beer MA. Discriminative prediction of mammalian enhancers from DNA sequence. Genome Res. 2011:21:2167-80.

12. Ghandi M, Lee D, Mohammad-Noori M, Beer MA. Enhanced regulatory sequence prediction using gapped k-mer features. PLoS Comput Biol. 2014;10:e1003711.

13. Visel A, Blow MJ, Li Z, Zhang T, Akiyama JA, Holt A, Plajzer-Frick I, Shoukry $M$, Wright C, Chen F, et al. ChIP-seq accurately predicts tissue-specific activity of enhancers. Nature. 2009;457:854-8.

14. Consortium EP. An integrated encyclopedia of DNA elements in the human genome. Nature. 2012;489:57-74.

15. Ernst J, Kheradpour P, Mikkelsen TS, Shoresh N, Ward LD, Epstein CB, Zhang X, Wang L, Issner R, Coyne M, et al. Mapping and analysis of chromatin state dynamics in nine human cell types. Nature. 2011:473:43-9.

16. Hoffman MM, Ernst J, Wilder SP, Kundaje A, Harris RS, Libbrecht M, Giardine B, Ellenbogen PM, Bilmes JA, Birney E, et al. Integrative annotation of chromatin elements from ENCODE data. Nucleic Acids Res. 2013;41:827-41

17. Ghandi M, Mohammad-Noori M, Ghareghani N, Lee D, Garraway L, Beer MA. gkmSVM: an R package for gapped-kmer SVM. Bioinformatics. 2016:32:2205-7.
18. Singh AP, Mishra S, Jabin S. Sequence based prediction of enhancer regions from DNA random walk. Sci Rep. 2018;8:15912.

19. Wang $X$, Lin $P$, Ho JWK. Discovery of cell-type specific DNA motif grammar in cis-regulatory elements using random Forest. BMC Genomics. 2018;19:929.

20. Weingarten-Gabbay S, Segal E. The grammar of transcriptional regulation Hum Genet. 2014;133:701-11.

21. Angermueller C, Parnamaa T, Parts L, Stegle O. Deep learning for computational biology. Mol Syst Biol. 2016;12:878

22. Quang D, Chen Y, Xie X. DANN: a deep learning approach for annotating the pathogenicity of genetic variants. Bioinformatics. 2015;31:761-3.

23. Zhou J, Troyanskaya OG. Predicting effects of noncoding variants with deep learning-based sequence model. Nat Methods. 2015;12:931-4.

24. Alipanahi B, Delong A, Weirauch MT, Frey BJ. Predicting the sequence specificities of DNA- and RNA-binding proteins by deep learning. Nat Biotechnol. 2015:33:831-8.

25. Liu F, Li H, Ren C, Bo X, Shu W. PEDLA: predicting enhancers with a deep learning-based algorithmic framework. Sci Rep. 2016;6:28517.

26. Min X, Zeng W, Chen S, Chen N, Chen T, Jiang R. Predicting enhancers with deep convolutional neural networks. BMC Bioinformatics. 2017;18:478.

27. Kleftogiannis D, Kalnis P, Bajic VB. DEEP: a general computational framework for predicting enhancers. Nucleic Acids Res. 2015;43:e6.

28. Quang D, Xie X. DanQ: a hybrid convolutional and recurrent deep neural network for quantifying the function of DNA sequences. Nucleic Acids Res. 2016;44:e107.

29. Tan KK, Le NQK, Yeh HY, Chua MCH. Ensemble of deep recurrent neural networks for identifying enhancers via dinucleotide physicochemical properties. Cells. 2019;8:767.

30. Ernst J, Kellis M. ChromHMM: automating chromatin-state discovery and characterization. Nat Methods. 2012:9:215-6.

\section{Publisher's Note}

Springer Nature remains neutral with regard to jurisdictional claims in published maps and institutional affiliations.
Ready to submit your research? Choose BMC and benefit from:

- fast, convenient online submission

- thorough peer review by experienced researchers in your field

- rapid publication on acceptance

- support for research data, including large and complex data types

- gold Open Access which fosters wider collaboration and increased citations

- maximum visibility for your research: over 100M website views per year

At BMC, research is always in progress.

Learn more biomedcentral.com/submissions 